\title{
3.11 MAGNETISM IN WHITE DWARFS
}

\author{
J. D. LANDSTREET* \\ Department of Astronomy, Columbia University, New York, N.Y., U.S.A. \\ and \\ J. R. P. ANGEL** \\ Columbia Astrophysics Laboratory, Columbia University, New York, N.Y., U.S.A.
}

\begin{abstract}
Searches have been made for the normal and quadratic Zeeman effect and broad-band circular polarization in white dwarf stars. A positive effect has been found in Grw $+70^{\circ} 8247$ whose continuum shows both linear and circular polarization.
\end{abstract}

It has been realized for some time that in gravitational collapse the magnetic flux passing through the star may be conserved, resulting in very high fields in the collapsed object (Woltjer, 1964). The discovery of pulsars, which are generally thought to be neutron stars with fields of order $10^{12} \mathrm{G}$, shows that magnetic flux must be largely conserved in collapse to a neutron star. If no flux is lost, the field strength is increased as $1 / R^{2}$, requiring initial fields before collapse of order $100 \mathrm{G}$. Such a value is not inconsistent with the fields observed in normal stars, ranging from about $1 \mathrm{G}$ in the sun to $10^{4} \mathrm{G}$ in magnetic A stars.

In collapse to a white dwarf, the reduction in radius is typically a factor of 100 , giving the possibility of field amplification of up to 10000 . Thus fields of between $10^{4}$ and $10^{8} \mathrm{G}$ could be expected, depending on the initial field. Various techniques exist to detect fields in this range. In the range from $10^{4}$ to $10^{6} \mathrm{G}$, the normal Zeeman effect is strong enough to be detectable, even though the spectral lines are very broad in dwarfs. We have searched for the Zeeman effect in the $\mathrm{H} \gamma$ absorption line of a dozen DA dwarfs, and have been able to set an upper limit of between $10^{4}$ and $10^{5} \mathrm{G}$ on their fields (Angel and Landstreet, 1970a). Above a few times $10^{5} \mathrm{G}$, the quadratic Zeeman effect would cause a detectable shift of spectral lines, as pointed out by Preston (1970). He concludes from the lack of such shifts that few if any white dwarfs with hydrogen absorption lines can have surface fields in excess of $5 \times 10^{5} \mathrm{G}$. Above a few times $10^{6} \mathrm{G}$ the quadratic Zeeman effect is so strong that spectral lines can be smeared out in a non-uniform field, or be so shifted as not to be identified. In this domain magnetic fields can be detected through the circular polarization of continuum radiation (Kemp et al., 1970a). It is this effect which has led to the recent discovery of a very strong field $\left(\sim 10^{7} \mathrm{G}\right)$ in one white dwarf, Grw $+70^{\circ} 8247$. So far this dwarf appears to be unique. A survey of other dwarfs without spectral lines has revealed no continuum circular polarization down to a level about two orders of magnitude less than that seen for Grw $+70^{\circ} 8247$ (Angel and Landstreet, 1970b).

* Present address: Department of Astronomy, University of Western Ontario, London, Ontario, Canada.

** Alfred P. Sloan Research Fellow. 
The initial observations of circular polarization of the continuum light of this star were made by Kemp at Oregon and confirmed by us at Kitt Peak, and are reported in the paper by Kemp et al. (1970b). Immediately after the discovery, we made extensive observations at the 107-in. telescope of McDonald Observatory to obtain the wavelength and time dependence of the effect, and discovered linear polarization. (Angel and Landstreet, 1970b) In brief, we find that the polarization is quite constant in time scale between a few seconds and a few days. The wavelength dependence is very striking: the component of circular polarization, which changes only slightly over the range of visible light, drops off very sharply in the ultraviolet, being less than $1 \%$ at $3300 \AA$. The linear polarization peaks in violet light and drops to zero in the red. Our detailed spectral measurements of both the linear and circular polarization below $7000 \AA$ agree to within a few percent with the later measurements in broader spectral bands by Gehrels. This agreement is very gratifying as the two techniques are quite different. The polarization measurements have been extended to the near infrared $(9200 \AA)$ by Gehrels, where the linear polarization reappears with position angle

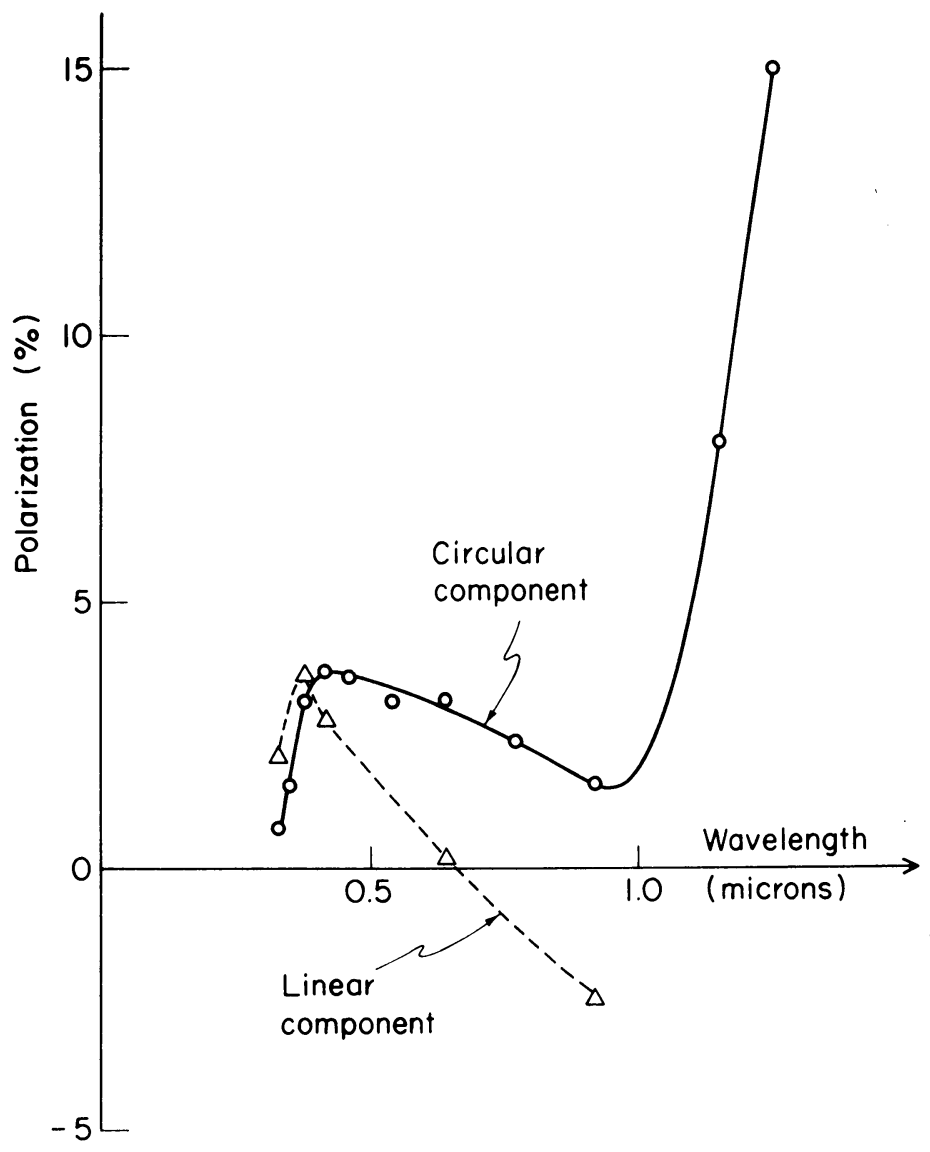

Fig. 1. Circular and linear components of polarization of $\mathrm{Grw}+70^{\circ} 8247$. 
rotated by about $90^{\circ}$, and to $1.2 \mu$ by Kemp, where the circular polarization increases very steeply. All the data so far are compiled on Figure 1.

The complex wavelength dependence of the polarization is not yet understood, but there seems little doubt that a strong magnetic field is responsible for the effect. The phenomenon of elliptical polarization by preferential absorption in a magnetic field is well known, and has been investigated in laboratory measurements of solids (the Faraday and Kerr magneto-optic effects) and in a thin plasma (propagation through the ionosphere). Elliptical polarization of emitted thermal radiation is the inverse process required by detailed balance and has been demonstrated in the laboratory for emission from hot solids and flames (Kemp et al., 1970a).

In the presence of a field, the opacity of the stellar atmosphere becomes polarization dependent, and in general will result in elliptical polarization. For the special case of propagation parallel to the magnetic field in an ionized medium, the absorption coefficients for the two senses of circular polarization are in the ratio $\left\{\left(\omega+\omega_{B}\right) /\left(\omega-\omega_{B}\right)\right\}^{2}$, where $\omega$ is the angular frequency of the propagated wave and $\omega_{B}$ is the cyclotron frequency $e B / m$ (Ratcliffe, 1959). This difference in opacity will lead to a net circular polarization $q$ of order $\omega_{B} / \omega$ in the continuum emission.

\section{Acknowledgements}

This work was supported by the Research Corporation, the Air Force Office of Scientific Research under Grant AFOSR-70-1945 and Contract 49(638)1358, and the National Aeronautics and Space Administration under Grants NGR-33-008-102 and NGR-33-008-012. It is Columbia Astrophysics Laboratory Contribution No. 30.

\section{References}

Angel, J. R. P. and Landstreet, J.: 1970a, Astrophys. J. Letters 160, L147.

Angel, J. R. P. and Landstreet, J.: 1970b, Astrophys. J. Letters 162, L61.

Kemp, J. C., Swedlund, J. B., and Evans, B. D.: 1970a, Phys. Rev. Letters 24, 1211.

Kemp, J. C., Swedlund, J. B., Landstreet, J., and Angel, J. R. P.: 1970b, Astrophys. J. Letters 161, L77.

Preston, G. W.: 1970, Astrophys. J. Letters 160, L143.

Ratcliffe, J. A.: 1959, The Magneto-Ionic Theory, Cambridge, England.

Woltjer, L.: 1964, Astrophys. J. 140, 1309.

\section{Discussion}

J. Kristian: Doesn't the observed wavelength dependence of the polarisation drastically disagree with Kemp's theory?

$R$. Angel: Yes, but it should not be taken to give accurately the effect in a white dwarf, where there is radiative transfer. There are many arguments, however, which lead one to believe that $V$ will be of order $\omega_{c} / \omega$, where $\omega_{c}$ is the cyclotron frequency. 